\title{
Stability Analysis of Periodic Orbits in a Class of Duffing-Like Piecewise Linear Vibrators
}

\author{
A. El Aroudi ${ }^{1, a}$, L. Benadero ${ }^{2}$, H. Ouakad ${ }^{3}$, and M. Younis ${ }^{4}$ \\ 1 Dept. of Electronics, Electrical Engineering and Automatic Control, Universitat Rovira i Virgili, Tarragona, Spain \\ 2 Dept. de Física Aplicada, Universitat Politècnica de Catalunya UPC, Barcelona, Spain \\ 3 Mechanical Engineering Department King Fahd University of Petroleum and Minerals, Dhahran, Saudi Arabia \\ 4 Physical Sciences and Engineering Division, King Abdullah University of Science and Technology, Saudi Arabia.
}

\begin{abstract}
In this paper, we study the dynamical behavior of a Duffing-like piecewise linear (PWL) springmass-damper system for vibration-based energy harvesting applications. First, we present a continuous time single degree of freedom PWL dynamical model of the system. From this PWL model, numerical simulations are carried out by computing frequency response and bifurcation diagram under a deterministic harmonic excitation for different sets of system parameter values. Stability analysis is performed using Floquet theory combined with Fillipov method.
\end{abstract}

\section{Introduction}

Vibration-based energy harvesters allow to convert the kinetic energy from a moving body due to ambient vibrations into electrical energy through a certain electromechanical mechanism. This growing area of research is driven by the capability of harvesters to act as an independent power supply for wireless microsystems and sensor networks, or, alternatively, to prolong the life time of batteries used in such systems. Energy harvesters can be modeled by single degree of freedom (SDOF) spring-mass systems. Vibrationbased energy harvesters consist of a mechanical resonator that exploit the ambient vibrations. Most of the studied energy harvesters are linear high quality factor ( $Q$ factor) SDOF resonators whose resonant frequency is designed to match the external excitation frequency with the aim to extract maximum power. In [1] a piecewise linear (PWL) system was proposed for vibration-based energy harvesting applications. The performance of the harvester when some suitable parameters vary were characterized by numerical simulations. In [2] this system was reconsidered and its dynamical behavior was studied by time-domain and frequency-domain numerical simulations using long-time integration performed on a continuous-time PWL model. The stability of the periodic orbits has been also addressed by using different approaches in [3]. Other studies dealing with PWL systems that can be used as energy harvesters can be found in [4], [5] where a prototype of an electromagnetic micro generator is designed and analyzed both numerically and experimentally. The aim of this paper is to present a study of the nonlinear behavior and stability analysis of a modified version of the system considered recently in [1] and [2]. The modification leads to a Duffinglike system with piecewise nonlinearity that exhibit periodic solutions whose stability can be studied by using Floquet theory and Fillipov method [6].

\footnotetext{
a e-mail: abdelali.elaroudi@urv.cat
}

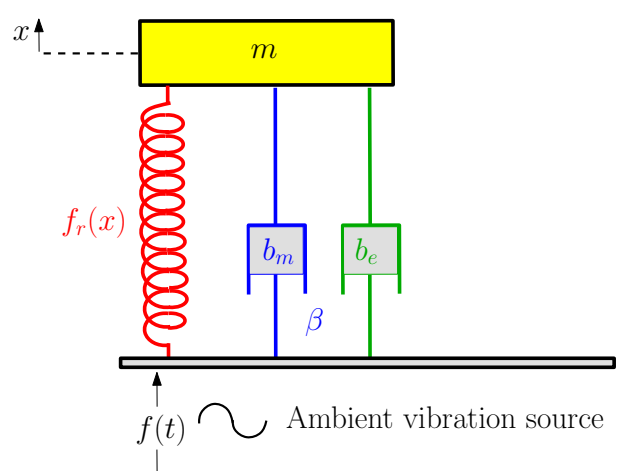

Fig. 1. Schematic diagram the spring-mass-damper system.

The rest of the paper is organized as follows. In Section 2, a brief description of the PWL system considered in this study is provided. Section 3 present a dimensionless formulation of the problem. Floquet theory with Fillipov method for switching PWL systems is reviewed in Section 4. In Section 5, numerical simulations illustrating bifurcation diagrams, frequency response and state-space trajectories are presented in Section 5 when some suitable system parameters are varied. The Floquet multipliers are calculated for different periodic orbits. Symmetry breaking or pitchfork, flip or period doubling and chaotic motion are shown to occur in the system. Finally, conclusions and discussions are presented in the last section.

\section{System description}

The schematic diagram of the system is shown in Fig. 1. It can be represented by a spring-mass-damper system where a piecewise linear (PWL) model for the stiffness is considered. The piecewise linearity comes from the restoring force $f_{r}(x)$ acting on the inertial mass which is assumed to 
have different stiffness values depending on the displacement with a linear term in each region. In particular, restoring force $f_{r}(x)$ is expressed as follows

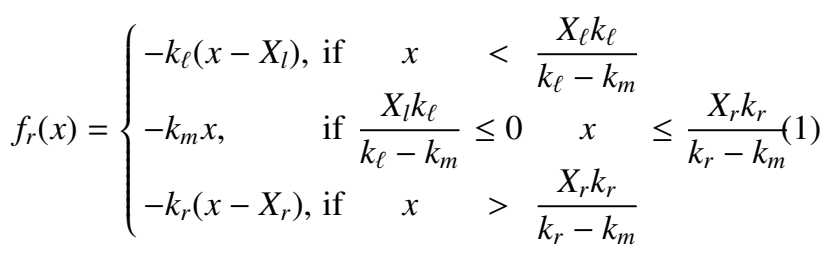

where $x$ is the mass displacement. In general $X_{r} \neq-X_{\ell}>$ $0, k_{\ell}>0, k_{r}>0$ and $k_{m}<0$. Let us consider that the system is driven with an external force $f(t)$. Therefore, the equation of the motion can be written as follows

$$
\ddot{x}+\frac{1}{m}\left(\beta \dot{x}+f_{r}(x)\right)=\frac{f(t)}{m}
$$

where the overdot stands for taking the derivative with respect to time, $\beta$ is the total damping which is the result of a mechanical damping $b_{m}$ and an electromechanical damping $b_{e}$. Let $y=\dot{x}$ be the velocity of the mass. Then, (2) can be written in the following form:

$$
\begin{aligned}
& \dot{x}=y \\
& \dot{y}=-\frac{1}{m}\left(\beta \dot{x}-+f_{r}(x)\right)+\frac{f(t)}{m}
\end{aligned}
$$

For simplicity in the study of bifurcation phenomena and stability analysis a simple sinusoidal vibrational $T$-periodic source is considered, i.e, $f(t)=F \sin \left(\omega_{f} t\right)$, where $T=$ $2 \pi / \omega_{f}$.

\section{Dimensionless formulation}

\subsection{Dimensionless parameters and state variables}

To reduce the number of bifurcation parameters let us define the following dimensionless parameters

$$
\begin{aligned}
\beta^{\prime} & =\frac{\beta}{m \omega_{0}}, \quad F^{\prime}=-\frac{F}{k_{\ell} X_{\ell}}, \omega_{f}^{\prime}=\frac{\omega_{f}}{\omega_{0}}, \\
X_{r}{ }^{\prime} & =-\frac{X_{r}}{X_{\ell}}, \quad k_{r}{ }^{\prime}=\frac{k_{r}}{k_{\ell}}, \quad k_{m}{ }^{\prime}=\frac{k_{m}}{k_{\ell}}
\end{aligned}
$$

where $\omega_{0}=\sqrt{k_{\ell} / m}$. This dimensionless formulation allows us to decrease the number of parameters from nine to six. Let us also define the following dimensionless state variables and time

$$
x^{\prime}=-\frac{x}{X_{\ell}}, \quad y^{\prime}=-\frac{y}{X_{\ell} \omega_{0}}, \quad t^{\prime}=\omega_{0} t
$$

For convenience of notation, in the following we drop the use of primes in the definition of dimensionless state variables and parameters. The dimensionless expression of the restoring force becomes

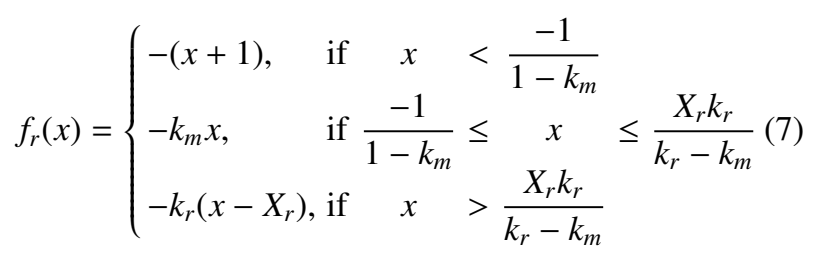

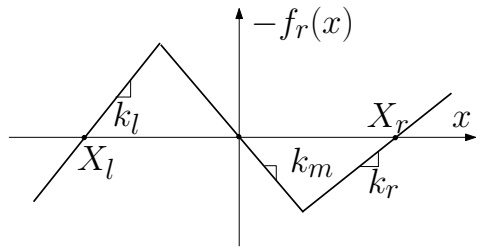

(a)

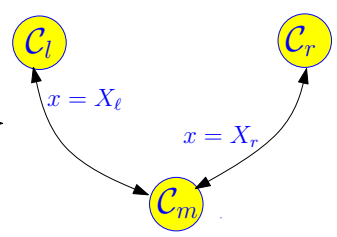

(b)
Fig. 2. Restoring force $f_{r}(x)$ (inverted) and transition state diagram between the different subsystems and the underlying conditions.

The dimensionless equation of the motion can be written as follows

$$
\begin{aligned}
& \dot{x}=y \\
& \dot{y}=-\beta \dot{x}+f_{r}(x)+f(t)
\end{aligned}
$$

Let us consider the symmetrical case where $k_{\ell}=k_{r}$ and $X_{\ell}=-X_{r}$. Under these conditions, the dimensionless equilibrium points are $X_{\ell}=-1$ and $X_{r}=1$ and the the dimensionless stiffness parameters are $k_{\ell}=1$ and $k_{r}=1$. The dynamics of the system is highly dependent on the system parameters. As the system is PWL, the dynamics for each system configuration can be described by a linear differential equation which can be written in the following form: $\dot{\mathbf{x}}=\mathbf{A}_{i} \mathbf{x}+\mathbf{B}_{i}(t):=\mathbf{f}_{i}(\mathbf{x}, t)$, where $\mathbf{x}=(x, y)$ is the system vector of state variables and where $i=1 \ldots 3$ is an index whose value depends on the configuration that the system takes. In all cases, $\mathbf{A}_{i}$ is a constant matrix while $\mathbf{B}_{i}(t)$ is a time varying periodic vector.

\subsection{Symmetry properties in the model}

Theorem 1 Let us consider the symmetrical case: $X_{\ell}=$ $-X_{r}$ and $k_{\ell}=k_{r}$. Let $\mathbf{x}_{1}(t)$ be solution of (8)-(9). Then, either $\mathbf{x}_{1}(t)$ is nonsymmetric and there exists another solution $\mathbf{x}_{2}(t)$ given by

$$
\mathbf{x}_{2}(t)=-\mathbf{x}_{1}\left(t \pm \frac{T}{2}\right)
$$

or $\mathbf{x}_{1}(t)$ is a unique symmetric solution with half period odd symmetry, i.e, $\mathbf{x}_{1}(t)=-\mathbf{x}_{1}\left(t \pm \frac{T}{2}\right)$.

Proof Let us write the model (8)-(9) as follows:

$$
\dot{\mathbf{x}}=\mathbf{f}(\mathbf{x}, t)
$$

The proof is straightforward by using (8)-(9) and observing that $\mathbf{f}\left(-\mathbf{x}, t \pm \frac{T}{2}\right)=\mathbf{f}(\mathbf{x}, t)$

\subsection{System linear configurations}

The possible transitions from one configuration to another and their different conditions are represented in Fig. 2 where three different possible configurations $\left(C_{1}, C_{2}\right.$ and $\left.C_{3}\right)$ that the system can take are shown. The linear equations corresponding to each configuration can be obtained straightforwardly from the system dynamical model (8)-(9). These configurations are 
- Configuration $1\left(C_{1}\right): f_{r}(x)=-(x+1)$. From (8)-(9), the system matrix $\mathbf{A}$ and vector $\mathbf{B}$ are

$$
\mathbf{A}_{1}=\left(\begin{array}{cc}
0 & 1 \\
-1 & -\beta
\end{array}\right), \quad \mathbf{B}_{1}=\left(\begin{array}{c}
0 \\
F \sin \left(\omega_{f} t\right)-1
\end{array}\right)
$$

- Configuration $2\left(C_{2}\right): f_{r}(x)=-k_{m} x$. From (8)-(9), the system matrix $\mathbf{A}$ and vector $\mathbf{B}$ are

$$
\mathbf{A}_{2}=\left(\begin{array}{cc}
0 & 1 \\
-k_{m} & -\beta
\end{array}\right), \quad \mathbf{B}_{2}=\left(\begin{array}{c}
0 \\
F \sin \left(\omega_{f} t\right)
\end{array}\right)
$$

- Configuration $3\left(C_{3}\right): f_{r}(x)=-k_{r}\left(x-X_{r}\right)$. From (8)-(9), the system matrix $\mathbf{A}$ and vector $\mathbf{B}$ are

$$
\mathbf{A}_{3}=\left(\begin{array}{cc}
0 & 1 \\
-k_{r} & -\beta
\end{array}\right), \quad \mathbf{B}_{3}=\left(\begin{array}{c}
0 \\
F \sin \left(\omega_{f} t\right)+k_{r} X_{r}
\end{array}\right)
$$

\section{Stability analysis using Floquet theory and Fillipov method}

An approach for stability analysis of piecewise linear systems is based on the Floquet theory and the eigenvalues of the monodromy matrix. The eigenvalues of this matrix are called Floquet multipliers. For piecewise linear systems, as is the case for the system considered in this study, the monodromy matrix can be constructed from the product of the state transition matrices corresponding to each sub-cycle and the corresponding saltation matrix [6]. Suppose a trajectory $\mathbf{x}(t)$ starts at time instant $t_{i}$ and is passing from the Configuration $C_{i}$ described by the vector field $\mathbf{A}_{i} \mathbf{x}+\mathbf{B}_{i}(t):=\mathbf{f}_{i}(\mathbf{x}, t)$, intersects the switching boundary described by the switching condition $\sigma_{i}(\mathbf{x}, t)=0$ at $t_{i}$, and goes to Configuration $C_{i+1}$ given by the vector field $\mathbf{A}_{i+1} \mathbf{x}+\mathbf{B}_{i+1}(t):=\mathbf{f}_{i+1}(\mathbf{x}, t)$. It has been shown, using the Fillipov method ([6]), that when there is a transversal intersection, the state transition matrix across the switching boundary, called also the saltation matrix $\mathbf{S}_{i}$, is given by

$$
\mathbf{S}_{i}=\mathbf{I}+\frac{\left(\mathbf{f}_{i+1}\left(\mathbf{x}\left(t_{i}\right)\right)-\mathbf{f}_{i}\left(\mathbf{x}\left(t_{i}\right)\right)\right) \mathbf{K}_{i}}{\mathbf{K}_{i} \mathbf{f}_{i}(\mathbf{x})+\left.\frac{\partial \sigma_{i}}{\partial t}\right|_{t=t_{i}}}
$$

where I is a unitary matrix with appropriate size. Then, the monodromy matrix $\mathbf{M}$ for a piecewise linear system with $N_{c}$ different configurations can be composed during a complete cycle as follows

$$
\mathbf{M}(\mathbf{x}(0))=\boldsymbol{\Phi}_{N_{c}} \mathbf{S}_{N_{c}-1} \ldots \boldsymbol{\Phi}_{2} \mathbf{S}_{1} \boldsymbol{\Phi}_{1}
$$

where $\boldsymbol{\Phi}_{i}=\boldsymbol{\Phi}_{i}\left(t_{i}-t_{i-1}\right)=e^{\mathbf{A}_{i}\left(t_{i}-t_{i-1}\right)}, i=1 \ldots N_{c}$, is the state transition matrix corresponding to the interval $\left(t_{i-1}, t_{i}\right)$ within which Configuration $C_{i}$ is taking place. It is worth noting that the previous approach can be applied to periodic orbits with any period.

\section{Numerical Results}

\subsection{Bifurcation behavior and stability analysis of periodic solutions}

The value of the dimensionless excitation frequency considered in this section is $\omega_{f}=0.3$. Bifurcation diagrams will be computed and bifurcation phenomena will be explained by using the Floquet multipliers.

\subsubsection{Case study $1: k_{m}=-0.5, \beta=0.2, F=1.0$}

Figure 3 shows the bifurcation diagram of the system by taking the frequency $\omega$ of the external force as a bifurcation parameter. The periodicity diagram and the Floquet multipliers of the periodic orbits are also plotted. It can be observed that away from the resonance the system exhibit stable periodic behavior. The system undergoes symmetry breaking bifurcation as the excitation frequency is increased from lower values than the resonant frequency or from higher values than the resonant frequency. Period doubling takes place in the vicinity of the resonant frequency. Successive period doubling may lead to chaotic behavior. This occur for $\omega \approx 1$. The corresponding Floquet multipliers and the period of the periodic orbits are plotted in Fig. 3(b) where both kinds of instabilities can be identified by observing the values of the Floquet multipliers at the critical points. Namely, at a symmetry breaking bifurcation, one of the Floquet multipliers is equal to 1 while at a period doubling bifurcation one of these multipliers is equal to -1 .

The frequency response of the system for different values of the force intensity is shown in Fig. 4. One can observe that the system frequency response is moved to low frequency regions for low values of external forcing amplitudes $F$. This could be an advantage for potential use of this system in low frequency vibrational-based energy harvesting.

Figure 5 shows time domain waveforms, and state-space trajectories for different values of the bifurcation parameter $\omega$. When more than one solution coexist, they are plotted together in the same diagram.

\subsubsection{Case study 2: $k_{m}=-0.5, \beta=0.2, \omega=0.3$}

Figure 6 shows the bifurcation diagram of the system by taking the amplitude $F$ of the external force as a bifurcation parameter for $\omega$ below the resonant frequency. The periodicity diagram and the Floquet multipliers of the periodic orbits are also shown in different panels of the figure. It can be observed that relatively very small values of $F$ and high values of this parameters leads the system to exhibit stable periodic behavior. The system undergoes symmetry breaking bifurcation as the excitation amplitude is increased from lower values while sudden emergence of chaotic behavior can be observed when the driving amplitude is decreased from high values to lower values. Windows of periodicity can be clearly observed. The corresponding Floquet multipliers and the period of the periodic orbits are plotted in Fig. 6(b). A symmetry breaking bifurcation can be observed in Fig. 6(c)where one of the Floquet multipliers is equal to 1 .

\subsubsection{Case study 3: $k_{m}=-0.5, \beta=0.2, \omega=1.1$}

Figure 7 shows the bifurcation diagram of the system by taking the amplitude $F$ of the external force as a bifurcation parameter for $\omega$ in the vicinity of the resonant frequency. The periodicity diagram and the Floquet multipliers of the periodic orbits are also shown. It can be observed the same bifurcation patterns as in the previous case are preserved but with less periodicity windows in this case. 

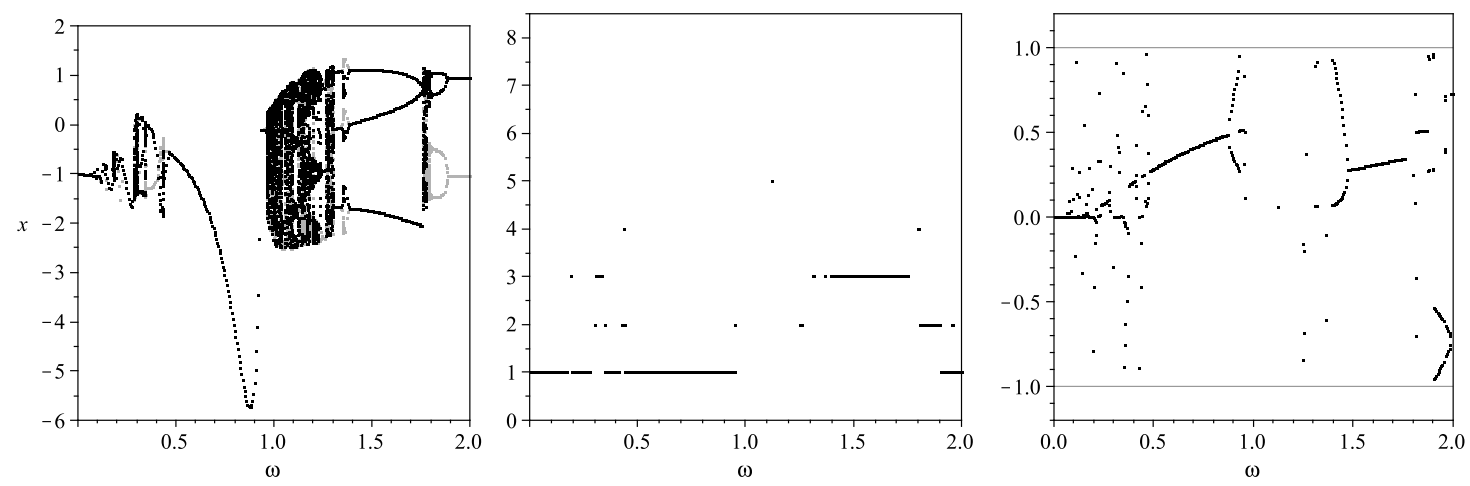

Fig. 3. Bifurcation diagrams, Floquet multipliers and fundamental period of the different stable periodic solutions of the PWL system taking $\omega$ as a bifurcation parameter for $k_{m}=-0.5$ and $\beta=0.2$ and $F=1.0$.

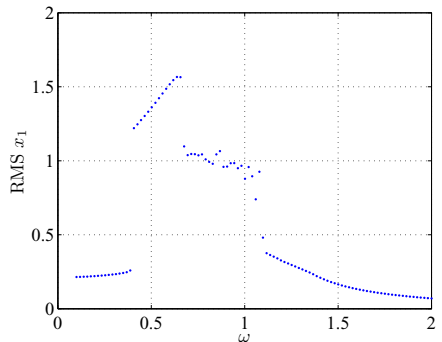

(a) $F=0.3$

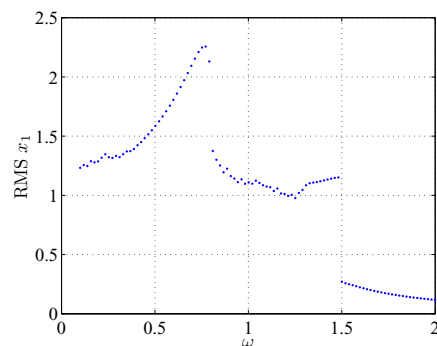

(b) $F=0.5$

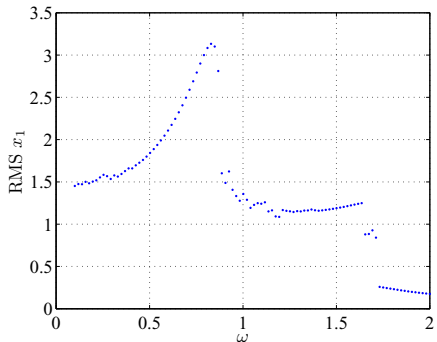

(c) $F=0.75$

Fig. 4. Different steady-state frequency response of the PWL system for different values of $F$ and for $k_{m}=-0.5$ and $\beta=0.2$.

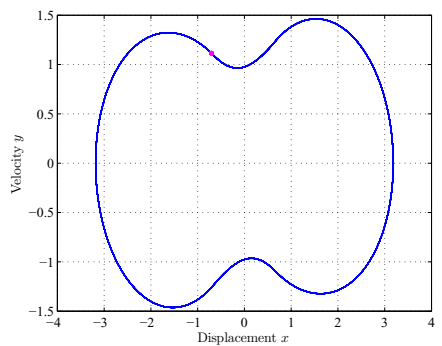

(a) $\omega=0.5$

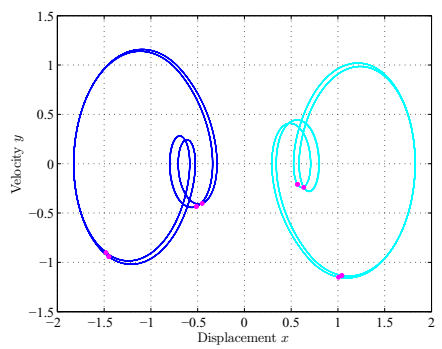

(d) $\omega=1.8$

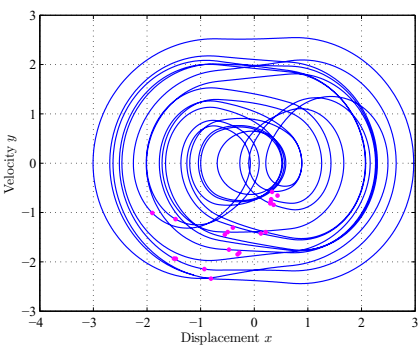

(b) $\omega=1$

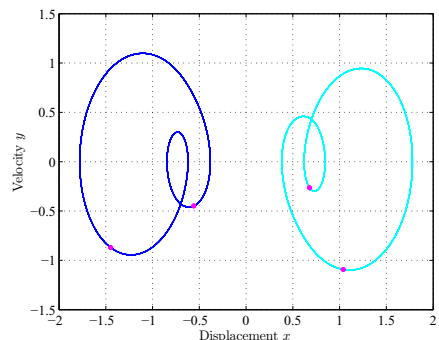

(e) $\omega=1.85$

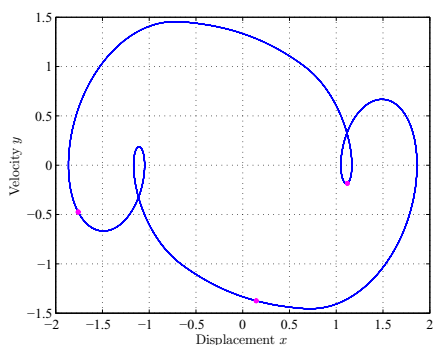

(c) $\omega=1.5$

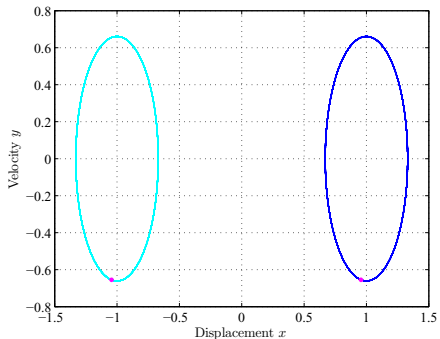

(f) $\omega=2$

Fig. 5. Different steady-state state-space trajectories of the PWL system for different values of $\omega$ and for $k_{m}=-0.5$ and $\beta=0.2$ and $F=1.0$. 

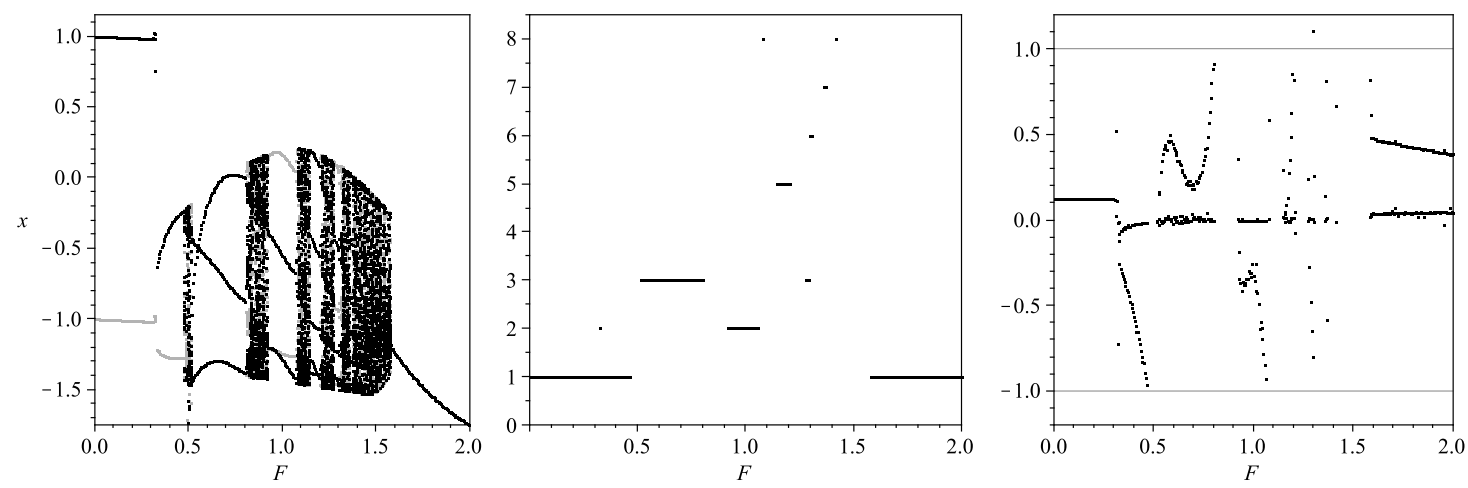

Fig. 6. Bifurcation diagrams, Floquet multipliers and fundamental period of the different stable periodic solutions of the PWL system taking $F$ as a bifurcation parameter for $k_{m}=-0.5, \beta=0.2, \omega=0.3$.
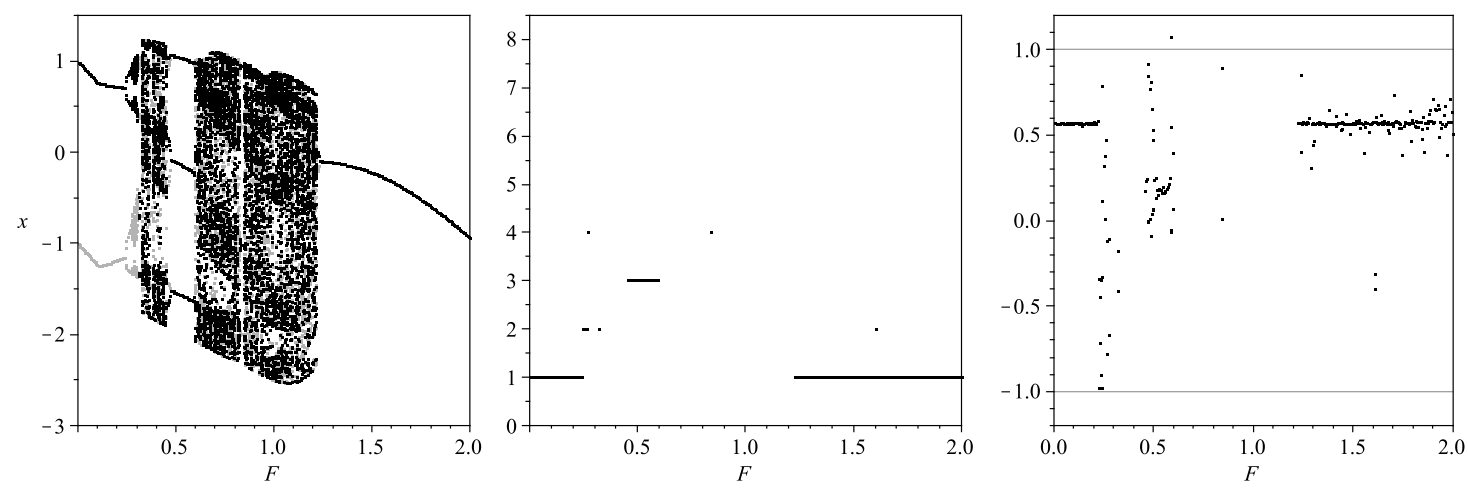

Fig. 7. Bifurcation diagrams, Floquet multipliers and fundamental period of the different stable periodic solutions of the PWL system taking $F$ as a bifurcation parameter for $k_{m}=-0.5, \beta=0.2, \omega=1.1$.

\section{Conclusions}

In this work, we studied the dynamics of a Duffing-like PWL spring-mass single degree of freedom system which can be used for vibration-based energy harvesting applications. The linear configurations corresponding to different regions of the state-space, where stiffness and restoring force take different values, were derived. The time-domain waveforms ad bifurcation diagrams were obtained from the PWL model revealing great variety of dynamical behavior such as symmetry breaking bifurcation, period doubling and chaotic regimes. Stability analysis of the PWL system was performed using Floquet theory with Fillipov method to deal with its switching nature.

\section{Acknowledgments}

This work was partially supported by the Ministerio de Ciencia e Innovación under grant CSD2009-00046.

\section{References}

1. Triplett A., Linton J. and Quinn D. D. "Electromagnetic Energy Harvesting," ENOC 2011, 24-29 July 2011, Rome, Italy.
2. El Aroudi A., Ouakad H. and Younis M. "Nonlinear Dynamics of A Piecewise Linear Energy Harvesting System", 1st Euro-Mediterranean Conference on Structural Dynamics and Vibroacoustics 23-25 Apr. 2013, Marrakech (Morocco).

3. El Aroudi A., Ouakad H., Benadero L. and Younis M. "Analysis of Bifurcation Behavior of a Piecewise Linear Vibrator for Energy Harvesting Applications", International Journal of Bifurcation and Chaos, in press, 2014.

4. Bendame M. and Abdel-Rahman E. "Nonlinear Modeling and Analysis of a Vertical Springless Energy Harvester", MATEC Web of Conferences 1, 01004 (2012), CSNDD 2011, Marrakech, Morocco, DOI: 10.1051/matecconf/20120101004, published by EDP Sciences, 2012.

5. Soliman M. S. M., Abdel-Rahman E. M., El-Saadany E. F. and Mansour R. R."A Wideband Vibrationbased Energy Harvester," J. Micromech. Microeng., 18, 115021 (11 pages), 2008.

6. Leine R. L. and Nijemeijer H. Dynamics and Bifurcations of Non-Smooth Mechanical Systems, Lecture Notes in Applied and Computational Mechanics, Springer, 2004. 